\title{
Preparation and NMR properties of derivatives of arylamino-methylidene malonic acid and pentane-2,4-dione
}

\author{
Denisa Tarabováa $^{a}$ Viktor Milata ${ }^{a}$, Jiří Hanusek ${ }^{b}$ \\ ${ }^{a}$ Faculty of Chemical and Food Technology, Institute of Organic Chemistry, Slovak University of Technology, \\ Radlinského street 9, SK-812 37 Bratislava, Slovak Republic \\ ${ }^{b}$ Faculty of Chemical Technology, Department of the Mechanisms of Organic Reactions, University of Pardubice, \\ Student street 95, CZ-532 10 Pardubice, Czech Republic \\ viktor.milata@stuba.sk
}

\begin{abstract}
A set of 22 anilinomethylidene derivatives (13 new ones) of di(m)ethyl malonate, malononitrile and pentane-2,4-dione with various substituents in position 4- and 3-, respectively were prepared to study the characteristic influence of these substituents and solvents on chemical shifts in 13C NMR spectra and like reference compounds for kinetic measurements.
\end{abstract}

Keywords: NMR spectroscopy, substituent chemical shifts (SCS), arylaminomethylidene derivatives, enamines

\section{Introduction}

Arylaminomethylidene malonic acid derivatives are very useful intermediates used in organic synthesis of quinolone derivatives (Hermecz et al. 1992). Most frequently are used esters, less frequently are dinitriles or their formal hybrids - cyanoacetates (Bella et al. 2012, Milata et al. 1987). Derivatives of 2,4-pentanedione (acetylacetone) are used rarely (Wolfbeis et al. 1977), but they are interesting from a point of view of physical chemists (Couchouron et al. 1983). We need the target compounds like standards for kinetic measurement with differently substituted anilines. From various synthetic approaches to target compounds the first step of Gould - Jacobs reaction: reaction of aniline with activated enolether is the most advantageous (Hermecz et al. 1992) (Fig. 1.).

\section{Materials and methods}

Aniline and 3, 4-substituted anilines respectively were distilled before use, 4-nitroaniline was recrystallized from 2-propanol (Fig. 1). Enolethers - dimethyl and diethyl methoxymethylidene malonates were commercially available (Sigma Aldrich ${ }^{\circledR}$ and Acros Organics $\left.{ }^{\circledR}\right)$. Commercially available ethoxymethylidene malononitrile was recrystallized from 2-propanol before use. Melting points were determined using Kofler hot plate without further corrections. Elemental analyses were determined using an automatic analysator Flash EA 1112 (fy Thermo Finnigan) and Fisons Instruments EA 1108 CHN.

IR spectra were taken on a FTIR Nicolet NEXUS 470 spectrophotometer using AT-R technique in region $3800-600 \mathrm{~cm}^{-1}$. UV-VIS measurements were<smiles>Nc1cc[c]cc1</smiles>

I

Ia: $\mathrm{Z}=\mathrm{H}$

Ib: $\mathrm{Z}=3-\mathrm{Cl}$;

Ic: $\mathrm{Z}=4-\mathrm{Br}$;

Id: $\mathrm{Z}=4-\mathrm{Me}$;

Ie: $\mathrm{Z}=4-\mathrm{OMe}$

If: $\mathrm{Z}=4-\mathrm{NO}_{2}$<smiles>[R]OC=C([Y])[X]</smiles>

II

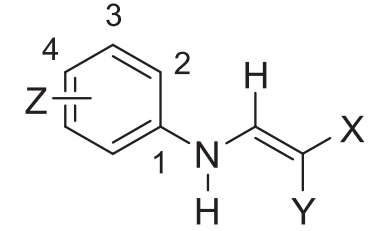

III

Fig. 1. Reaction of anilines with enolethers. 
recorded on UV-VIS NIR 3600 SHIMADZU spectrophotometer in region $250-700 \mathrm{~nm} .{ }^{1} \mathrm{H}$ and ${ }^{13} \mathrm{C}$ NMR spectra were measured in $\mathrm{CDCl}_{3}$ and DMSO- $d_{6}$ solution using spectrophotometers Varian VXR-300 (for ${ }^{1} \mathrm{H}$ 299,995 MHz, for ${ }^{13} \mathrm{C} 75,431 \mathrm{MHz}$ ) and Bruker Avance III 400 (for ${ }^{1} \mathrm{H} 400,23 \mathrm{MHz}$, for ${ }^{13} \mathrm{C} 100,65$ $\mathrm{MHz})$ at $25^{\circ} \mathrm{C}$. Chemical shifts $(\delta$-scale) are quoted in parts per million and following abbreviations are used: $\mathrm{s}=$ singlet; $\mathrm{d}=$ doublet; $\mathrm{t}=$ triplet; $\mathrm{q}=$ quartet, $\mathrm{m}=$ multiplet.

\section{Experimental}

Preparation of the title compounds was realized according to Fig. 1.

\section{General procedure for preparation of arylaminomethylidene derivatives III}

Suitable substituted or nonsubstituted aniline Ia-f (5 mmol) dissolved in $\mathrm{MeOH} / \mathrm{EtOH}(15-20 \mathrm{ml})$ was mixed with equimolar amount of enolether IIa-d $(5 \mathrm{mmol})$ in $\mathrm{MeOH} / \mathrm{EtOH}(15-20 \mathrm{ml})$. Reaction mixture was stirred at $\mathrm{rt}$ from $15 \mathrm{~min}$ up to $3 \mathrm{~h}$ at reflux. After end of the reaction solvent was evaporated through vacuum evaporator. Thus obtained raw products were purified by column chromatography or recrystallization. Purification and individual reaction conditions for all compounds are presented.

\section{Dimethyl 2-l(phenylamino)methylidene/malonate (IIIa, $Z=H$ )}

There was used to a reaction aniline $(\mathbf{I a}, \mathbf{Z}=\mathbf{H})$ $(0.47 \mathrm{~g}, 5 \mathrm{mmol})$ in $10 \mathrm{ml} \mathrm{MeOH}$ and enolether IIa $(0.87 \mathrm{~g}, 5 \mathrm{mmol})$ in $15 \mathrm{ml} \mathrm{MeOH}$. Reaction mixture was stirred $20 \mathrm{~min}$ at $\mathrm{rt}$, then solvent was evaporated. Raw product was purified by column chromatography (EA (ethyl acetate):H(hexane) $=1: 1$, $\left.\mathrm{R}_{\mathrm{F}}=0.24\right)$.

Yield: $1.02 \mathrm{~g}, 87 \%$, light yellow solid. Mp $46-48^{\circ} \mathrm{C}$ $\left(47^{\circ} \mathrm{C}\right.$, Rappoport et al. 1972).

${ }^{1} \mathbf{H}$ NMR (300 MHz, $\mathrm{CDCl}_{3}$ ) $\delta$ (ppm) 11.01 (br d, $\left.1 \mathrm{H},{ }^{3} J=13.1 \mathrm{~Hz},-\mathrm{NH}\right), 8.54\left(\mathrm{~d}, 1 \mathrm{H},{ }^{3} J=13.8 \mathrm{~Hz}\right.$, $-\mathrm{CH}=), 7.41-7.36(\mathrm{~m}, 2 \mathrm{H}), 7.20-7.14(\mathrm{~m}, 3 \mathrm{H})$, 3.87 (s, 3H, OMe), 3.78 (s, 3H, OMe)

UV VIS (DMSO, nm) $\lambda_{\max } 319$

Elemental analysis for $\mathrm{C}_{12} \mathrm{H}_{13} \mathrm{NO}_{4}$ - calculated (found) (\%): C 61.27 (61.25), H 5.57 (5.52), N $5.95(5.91)$

\section{Diethyl 2-[(phenylamino)methylidene]malonate}

$($ IIIb, $Z=H)$

There was used to a reaction aniline $(\mathbf{I a}, \mathbf{Z}=\mathbf{H})$ $(0.47 \mathrm{~g}, 5 \mathrm{mmol})$ in $10 \mathrm{ml} \mathrm{EtOH}$ and enolether IIb (1.1 g, $5 \mathrm{mmol}$ ) in $15 \mathrm{ml} \mathrm{EtOH}$. Reaction mixture was stirred $20 \mathrm{~min}$ at $\mathrm{rt}$, then solvent was evaporated.
Raw product was purified by column chromatography. (EA:H $=1: 1, \mathrm{R}_{\mathrm{F}}=0.29$ ).

Yield: $1.25 \mathrm{~g}, 93 \%$, light yellow solid. Mp 51-53 ${ }^{\circ} \mathrm{C}$ (54-55 ${ }^{\circ} \mathrm{C}$, Huppatz et al. 1981).

${ }^{1}$ H NMR (300 MHz, $\mathrm{CDCl}_{3}$ ) $\delta$ (ppm) 11.01 (br d, $\left.1 \mathrm{H},{ }^{3} \mathrm{~J}=13.5 \mathrm{~Hz},-\mathrm{NH}\right), 8.53\left(\mathrm{~d}, 1 \mathrm{H},{ }^{3} \mathrm{~J}=13.7 \mathrm{~Hz}\right.$, $-\mathrm{CH}=), 7.38(\mathrm{~m}, 2 \mathrm{H}), 7.16(\mathrm{~m}, 3 \mathrm{H}), 4.32(\mathrm{q}, 2 \mathrm{H}$, $\left.{ }^{3} J=7.1 \mathrm{~Hz}, \mathrm{OCH}_{2}\right), 4.25\left(\mathrm{q}, 2 \mathrm{H},{ }^{3} \mathrm{~J}=7.1 \mathrm{~Hz}, \mathrm{OCH}_{2}\right)$, $1.39\left(\mathrm{t}, 3 \mathrm{H},{ }^{3} \mathrm{~J}=7.1 \mathrm{~Hz}, \mathrm{CH}_{3}\right), 1.33\left(\mathrm{t}, 3 \mathrm{H},{ }^{3} \mathrm{~J}=7.1 \mathrm{~Hz}\right.$, $\mathrm{CH}_{3}$ )

UV VIS (DMSO, nm) $\lambda_{\max } 321$

\section{3-[(Phenylamino $)$ methylidene]pentane-2,4-dione}

(IIIC, $Z=H$ )

There was used to a reaction aniline $(\mathbf{I a}, \mathbf{Z}=\mathbf{H})$ $(0.47 \mathrm{~g}, 5 \mathrm{mmol})$ in $10 \mathrm{ml} \mathrm{EtOH}$ and enolether IIc $(0.78 \mathrm{~g}, 5 \mathrm{mmol})$ in $15 \mathrm{ml} \mathrm{EtOH}$. Reaction mixture was stirred $15 \mathrm{~min}$ at $\mathrm{rt}$ and solvent was evaporated. Raw product was purified by column chromatography $\left(\mathrm{EA}: \mathrm{H}=1: 1, \mathrm{R}_{\mathrm{F}}=0.43\right)$.

Yield: $0.94 \mathrm{~g}, 94 \%$, yellow solid. Mp 85-87 ${ }^{\circ} \mathrm{C}$ $\left(90{ }^{\circ} \mathrm{C}\right.$, Wolfbeis et al. 1979).

${ }^{1} \mathbf{H}$ NMR (300 MHz, $\mathrm{CDCl}_{3}$ ) $\delta$ (ppm) 12.70 (br s, $1 \mathrm{H},-\mathrm{NH}), 8.19\left(\mathrm{~d}, 1 \mathrm{H},{ }^{3} \mathrm{~J}=12.8 \mathrm{~Hz},-\mathrm{CH}=\right)$, $7.38-7.33(\mathrm{~m}, 2 \mathrm{H}), 7.19-7.11(\mathrm{~m}, 3 \mathrm{H}), 2.50(\mathrm{~s}, 3 \mathrm{H}$, $\mathrm{Me}), 2.33$ (s, 3H, Me)

UV VIS (DMSO, nm) $\lambda_{\max } 330$

Elemental analysis for $\mathrm{C}_{12} \mathrm{H}_{13} \mathrm{NO}_{2}$ - calculated (found) (\%): C 70.92 (70.77), H 6.45 (6.33), N $6.89(6.78)$

\section{2-[(Phenylamino $)$ methylidene]malononitrile (IIId, $Z=H$ )}

There was used to a reaction aniline $(\mathbf{I} \mathbf{a}, \mathbf{Z}=\mathbf{H})$ $(0.47 \mathrm{~g}, 5 \mathrm{mmol})$ in $10 \mathrm{ml} \mathrm{EtOH}$ and enolether IId $(0.61 \mathrm{~g}, 5 \mathrm{mmol})$ in $15 \mathrm{ml} \mathrm{EtOH}$. Reaction mixture was stirred $20 \mathrm{~min}$ at $\mathrm{rt}$ and solvent was evaporated. Raw product was purified by column chromatography $\left(\mathrm{EA}: \mathrm{H}=1: 1, \mathrm{R}_{\mathrm{F}}=0.15\right)$.

Yield: $0.74 \mathrm{~g}, 88 \%$, yellow powder. Mp $248-249^{\circ} \mathrm{C}$ (254-256 ${ }^{\circ} \mathrm{C}$, Nasakin et al. 1992).

${ }^{1}$ H NMR (300 MHz, DMSO- $d_{6}$ ) $\delta$ (ppm) 11.1 (br d, $1 \mathrm{H},-\mathrm{NH}), 8.51(\mathrm{~s}, 1 \mathrm{H},-\mathrm{CH}=), 7.46-7.36(\mathrm{~m}$, $4 \mathrm{H}), 7.20-7.16(\mathrm{~m}, 1 \mathrm{H})$

UV VIS (DMSO, nm) $\lambda_{\max } 317$

Elemental analysis for $\mathrm{C}_{10} \mathrm{H}_{7} \mathrm{~N}_{3}-$ calculated (measured) (\%): C 70.99 (70.86), H 4.17 (4.06), N $24.84(24.91)$

Dimethyl 2-[(3-chlorophenylamino)methylidenelmalonate (IIIa, $\mathrm{Z}=3-\mathrm{Cl}$ )

There was used to a reaction 3-chloroaniline (Ib, $\mathbf{Z}=\mathbf{3 - C l})(0.64 \mathrm{~g}, 5 \mathrm{mmol})$ in $10 \mathrm{ml} \mathrm{MeOH}$ and enolether IIa $(0.87 \mathrm{~g}, 5 \mathrm{mmol})$ in $20 \mathrm{ml} \mathrm{MeOH}$. Reaction mixture was refluxed under regular stirring for $2 \mathrm{~h}$. After its cooling solvent was evaporated and a raw 
product was purified by column chromatography. (EA:H $\left.=1: 1, \mathrm{R}_{\mathrm{F}}=0.35\right)$.

Yield: $1.21 \mathrm{~g}, 90 \%$, white powder. Mp $146-147^{\circ} \mathrm{C}$. ${ }^{1} \mathbf{H}$ NMR $\left(300 \mathrm{MHz}^{\mathrm{CDCl}}{ }_{3}\right) \delta(\mathrm{ppm}) 11.01(\mathrm{~d}, 1 \mathrm{H}$, $\left.{ }^{3} J=13.8 \mathrm{~Hz},-\mathrm{NH}\right), 8.47\left(\mathrm{~d}, 1 \mathrm{H},{ }^{3} \mathrm{~J}=13.6 \mathrm{~Hz}\right.$, $-\mathrm{CH}=)$, 7.32-7.11 (m, 3H), 7.03-7.01 (m, 1H), $3.86(\mathrm{~s}, 3 \mathrm{H}, \mathrm{OMe}), 3.78(\mathrm{~s}, 3 \mathrm{H}, \mathrm{OMe})$

IR $\left(v, \mathrm{~cm}^{-1}\right) 2954,1588,1573,1231,769$

UV VIS (DMSO, nm) $\lambda_{\max } 321$

Elemental analysis for $\mathrm{C}_{12} \mathrm{H}_{12} \mathrm{ClNO}_{4}$ calculated (found) (\%): C 53.44 (53.24), H 4.49 (4.48), N 5.19 (4.96)

Diethyl 2-[(3-chlorophenylamino)methylidene]malonate (IIIb, $Z=3-C l)$

There was used to a reaction 3-chloroaniline (I Ib, $\mathbf{Z}=\mathbf{3 - C l})(0.64 \mathrm{~g}, 5 \mathrm{mmol})$ in $15 \mathrm{ml} \mathrm{EtOH}$ and enolether IIb (1.1 g, $5 \mathrm{mmol})$ in $20 \mathrm{ml} \mathrm{EtOH}$. Reaction mixture was refluxed under regular stirring for $2 \mathrm{~h}$. After its cooling solvent was evaporated and a raw product was purified by column chromatography (EA: $\left.\mathrm{H}=1: 1, \mathrm{R}_{\mathrm{F}}=0.40\right)$.

Yield: $1.44 \mathrm{~g}, 95 \%$, colourless solid. Mp $41-44^{\circ} \mathrm{C}$ (44-45 ${ }^{\circ} \mathrm{C}$, Fodor et al. 1948).

${ }^{1} \mathbf{H}$ NMR (300 MHz, $\left.\mathrm{CDCl}_{3}\right) \delta$ (ppm) $10.98(\mathrm{~d}, 1 \mathrm{H}$, $\left.{ }^{3} J=13.3 \mathrm{~Hz},-\mathrm{NH}\right), 8.46\left(\mathrm{~d}, 1 \mathrm{H},{ }^{3} \mathrm{~J}=13.5 \mathrm{~Hz}\right.$, $-\mathrm{CH}=), \quad 7.33-7.28(\mathrm{~m}, 1 \mathrm{H}), 7.15-7.11 \quad(\mathrm{~m}$, 2H), 7.03-7.00 (m, 1H), $4.32\left(\mathrm{q}, 2 \mathrm{H},{ }^{3} \mathrm{~J}=7.2 \mathrm{~Hz}\right.$, $\left.\mathrm{OCH}_{2}\right), 4.26\left(\mathrm{q}, 2 \mathrm{H},{ }^{3} \mathrm{~J}=7.1 \mathrm{~Hz}, \mathrm{OCH}_{2}\right), 1.39(\mathrm{t}, 3 \mathrm{H}$, $\left.{ }^{3} J=7.1 \mathrm{~Hz}, \mathrm{CH}_{3}\right), 1.35\left(\mathrm{t}, 3 \mathrm{H},{ }^{3} J=7.1 \mathrm{~Hz}, \mathrm{CH}_{3}\right)$ UV VIS (DMSO, nm) $\lambda_{\text {max }} 300$

3-[(3-Chlorophenylamino)methylidene]pentane-2, 4-dione (IIIc, $Z=3-C l$ )

There was used to a reaction 3-chloroaniline (I $\mathbf{l b}$, $\mathbf{Z}=3-\mathbf{C l})(0.64 \mathrm{~g}, 5 \mathrm{mmol})$ in $10 \mathrm{ml} \mathrm{EtOH}$ and enolether IIc $(0.79 \mathrm{~g}, 5 \mathrm{mmol})$ in $20 \mathrm{ml} \mathrm{EtOH}$. Reaction mixture was refluxed under regular stirring for $1 \mathrm{~h}$. After its cooling solvent was evaporated and a raw product was purified by column chromatography (EA:H $\left.=1: 1, \mathrm{R}_{\mathrm{F}}=0.22\right)$.

Yield: $1.16 \mathrm{~g}, 98 \%$, light yellow solid. Mp 89-91 ${ }^{\circ} \mathrm{C}$ (92-94 ${ }^{\circ} \mathrm{C}$, Snyder 1946).

${ }^{1}$ H NMR $\left(300 \mathrm{MHz}, \mathrm{DMSO}-d_{6}\right) \delta(\mathrm{ppm}) 12.43(\mathrm{~d}$, $\left.1 \mathrm{H},{ }^{3} \mathrm{~J}=12.7 \mathrm{~Hz},-\mathrm{NH}\right), 8.40\left(\mathrm{~d}, 1 \mathrm{H},{ }^{3} \mathrm{~J}=12.7 \mathrm{~Hz}\right.$, $-\mathrm{CH}=), 7.73-7.40(\mathrm{~m}, 3 \mathrm{H}), 7.25-7.21(\mathrm{~m}, 1 \mathrm{H})$, $2.39(\mathrm{~s}, 6 \mathrm{H}, 2 \times \mathrm{Me})$

UV VIS (DMSO, nm) $\lambda_{\max } 342$

IR $\left(v, \mathrm{~cm}^{-1}\right) 3057,1589,1567,1306,778$

Elemental analysis for $\mathrm{C}_{12} \mathrm{H}_{12} \mathrm{ClNO}_{2}$ - calculated (found) (\%): C 60.64 (60.70), H 5.09 (5.07), N $5.89(5.71)$

\section{2-[(3-Chlorophenylamino)methylidene]malononitrile} (IIId, $Z=3-C l)$

There was used to a reaction 3-chloroaniline (Ib,
$\mathbf{Z}=$ 3-Cl) $(0.64 \mathrm{~g}, 5 \mathrm{mmol})$ in $10 \mathrm{ml} \mathrm{EtOH}$ and enolether IId (0.61 g, $5 \mathrm{mmol})$ in $15 \mathrm{ml}$ EtOH. Reaction mixture was refluxed under regular stirring for $1 \mathrm{~h}$. After its cooling solvent was evaporated and a raw product was purified by column chromatography (EA:H $\left.=1: 1, \mathrm{R}_{\mathrm{F}}=0.28\right)$.

Yield: $0.96 \mathrm{~g}, 90 \%$, colourless solid. Mp $199-200{ }^{\circ} \mathrm{C}$ (200-201 ${ }^{\circ} \mathrm{C}$, Santilli 1964).

${ }^{1}$ H NMR (300 MHz, DMSO- $\left.d_{6}\right) \delta(\mathrm{ppm}) 11.16(\mathrm{br}$ $\mathrm{s}, 1 \mathrm{H},-\mathrm{NH}), 8.57(\mathrm{~s}, 1 \mathrm{H},-\mathrm{CH}=), 7.59(\mathrm{~s}, 1 \mathrm{H})$, $7.40-7.20(\mathrm{~m}, 3 \mathrm{H})$

UV VIS (DMSO, nm) $\lambda_{\max } 325$

IR $\left(v, \mathrm{~cm}^{-1}\right)$ 3203, 2227, 2216, 1662, 1338, 772

Elemental analysis for $\mathrm{C}_{10} \mathrm{H}_{6} \mathrm{ClN}_{3}$ - calculated (found) (\%): C 58.98 (58.84), H 2.97 (2.94), N $20.64(20.51)$

Dimethyl 2-[(4-bromophenylamino)methylidene]malonate (IIIa, $\mathrm{Z}=4-\mathrm{Br}$ )

There was used to a reaction 4-bromoaniline (Ic, $\mathbf{Z}=4$-Br $)(0.86 \mathrm{~g}, 5 \mathrm{mmol})$ in $15 \mathrm{ml} \mathrm{MeOH}$ and enolether IIa $(0.87 \mathrm{~g}, 5 \mathrm{mmol})$ in $20 \mathrm{ml} \mathrm{MeOH}$. Reaction mixture was refluxed under regular stirring for $1.5 \mathrm{~h}$. After its cooling raw product was collected by filtration and washed off with cold $\mathrm{MeOH}$.

Yield: $1.15 \mathrm{~g}, 73 \%$, white powder. Mp 113-114 ${ }^{\circ} \mathrm{C}$ (116-117 ${ }^{\circ} \mathrm{C}$, Rappoport et al. 1972).

${ }^{1}$ H NMR (300 MHz, DMSO- $\left.d_{6}\right) \delta(p p m) 10.66(\mathrm{~s}$, $1 \mathrm{H},-\mathrm{NH}), 8.38$ (br s, $1 \mathrm{H},-\mathrm{CH}=), 7.55(\mathrm{~d}, 2 \mathrm{H}$, $\left.{ }^{3} J=8.9 \mathrm{~Hz}, \mathrm{H}-3, \mathrm{H}-5\right), 7.37$ (d, 2H, ${ }^{3} \mathrm{~J}=8.9 \mathrm{~Hz}, \mathrm{H}-2$, H-6), 3.72 (s, 3H, OMe), 3.66 (s, 3H, OMe)

UV VIS (DMSO, nm) $\lambda_{\max } 328$

Elemental analysis for $\mathrm{C}_{12} \mathrm{H}_{12} \mathrm{BrNO}_{4}-$ calculated (found) (\%): C 45.88 (45.94), H 3.85 (3.82), N 4.46 (4.28)

Diethyl 2-[(4-bromophenylamino)methylidene]malonate (IIIb, $Z=4-B r$ )

There was used to a reaction 4-bromoaniline (Ic, $\mathbf{Z}=\mathbf{4 - B r})(0.86 \mathrm{~g}, 5 \mathrm{mmol})$ in $15 \mathrm{ml} \mathrm{EtOH}$ and enolether IIb (1.1 g, $5 \mathrm{mmol})$ in $20 \mathrm{ml} \mathrm{EtOH}$. Reaction mixture was refluxed under regular stirring for $1.5 \mathrm{~h}$. After its cooling raw product was purified by column chromatography.

Yield: $1.60 \mathrm{~g}, 92 \%$, colourless solid. Mp 98-99 ${ }^{\circ} \mathrm{C}$ (100.5-101.7 ${ }^{\circ} \mathrm{C}$, Lopez et al. 2010).

${ }^{1} \mathbf{H}$ NMR $\left(300 \mathrm{MHz}, \mathrm{DMSO}-d_{6}\right) \delta(\mathrm{ppm}) 10.65(\mathrm{~d}$, $\left.1 \mathrm{H},{ }^{3} \mathrm{~J}=13.8 \mathrm{~Hz},-\mathrm{NH}\right), 8.34\left(\mathrm{~d}, 1 \mathrm{H},{ }^{3} \mathrm{~J}=13.8 \mathrm{~Hz}\right.$, $-\mathrm{CH}=), 7.54\left(\mathrm{~d}, 2 \mathrm{H},{ }^{3} \mathrm{~J}=8.8 \mathrm{~Hz}, \mathrm{H}-3, \mathrm{H}-5\right), 7.34(\mathrm{~d}$, $\left.2 \mathrm{H},{ }^{3} \mathrm{~J}=8.9 \mathrm{~Hz}, \mathrm{H}-2, \mathrm{H}-6\right), 4.19$ (q, 2H, ${ }^{3} \mathrm{~J}=7.1 \mathrm{~Hz}$, $\left.\mathrm{OCH}_{2}\right), 4.11\left(\mathrm{q}, 2 \mathrm{H},{ }^{3} \mathrm{~J}=7.1 \mathrm{~Hz}, \mathrm{OCH}_{2}\right), 1.25(\mathrm{t}, 3 \mathrm{H}$, $\left.{ }^{3} J=7.1 \mathrm{~Hz}, \mathrm{CH}_{3}\right), 1.21\left(\mathrm{t}, 3 \mathrm{H},{ }^{3} J=7.1 \mathrm{~Hz}, \mathrm{CH}_{3}\right)$

UV VIS (DMSO, nm) $\lambda_{\max } 323$

Elemental analysis for $\mathrm{C}_{14} \mathrm{H}_{16} \mathrm{BrNO}_{4}-$ calculated (found) (\%): C 49.14 (49.20), H 4.71 (4.77), N $4.09(3.91)$ 
3-[(4-Bromophenylamino)methylidenelpentane-2, 4-dione (IIIc, $\mathrm{Z}=4-\mathrm{Br}$ )

There was used to a reaction 4-bromoaniline (Ic, $\mathbf{Z}=4$-Br $)(0.86 \mathrm{~g}, 5 \mathrm{mmol})$ in $10 \mathrm{ml} \mathrm{EtOH}$ and enolether IIc $(0.79 \mathrm{~g}, 5 \mathrm{mmol})$ in $20 \mathrm{ml} \mathrm{EtOH}$. Reaction mixture was stirred for $30 \mathrm{~min}$ at $\mathrm{rt}$. Solvent was evaporated and a raw product was collected by filtration and finally washed off with cold EtOH.

Yield: $1.30 \mathrm{~g}, 91 \%$, colourless crystals. Mp 139$140^{\circ} \mathrm{C}$.

${ }^{1} \mathbf{H}$ NMR $\left(300 \mathrm{MHz}, \mathrm{CDCl}_{3}\right) \delta(\mathrm{ppm}) 12.72(\mathrm{br} \mathrm{s}, 1 \mathrm{H}$, $-\mathrm{NH}), 8.16\left(\mathrm{~d}, 1 \mathrm{H},{ }^{3} \mathrm{~J}=12.6 \mathrm{~Hz},-\mathrm{CH}=\right), 7.50(\mathrm{~d}$, $\left.2 \mathrm{H},{ }^{3} \mathrm{~J}=8.7 \mathrm{~Hz}, \mathrm{H}-3, \mathrm{H}-5\right), 7.05\left(\mathrm{~d}, 2 \mathrm{H},{ }^{3} \mathrm{~J}=8.7 \mathrm{~Hz}\right.$, H-2, H-6), 2.54 (s, 3H, Me), 2.38 (s, 3H, Me)

UV VIS (DMSO, nm) $\lambda_{\max } 338$

Elemental analysis for $\mathrm{C}_{12} \mathrm{H}_{12} \mathrm{BrNO}_{2}$ - calculated (found) (\%): C 51.09 (51.00), H 4.29 (4.18), N $4.96(4.89)$

\section{2-[(4-Bromophenylamino)methylidene]malononitrile (IIId, $Z=4-B r)$}

There was used to a reaction 4-bromoaniline (Ic, $\mathbf{Z}=\mathbf{4 - B r})(0.86 \mathrm{~g}, 5 \mathrm{mmol})$ in $15 \mathrm{ml} \mathrm{EtOH}$ and enolether IId (0.61 g, $5 \mathrm{mmol})$ in $20 \mathrm{ml} \mathrm{MeOH}$. Reaction mixture was stirred for $30 \mathrm{~min}$ at $\mathrm{rt}$. Solvent was evaporated and a raw product was collected by filtration and finally washed with cold EtOH.

Yield: $0.69 \mathrm{~g}, 56 \%$, colourless solid. Mp 251-253 ${ }^{\circ} \mathrm{C}$.

${ }^{1} \mathbf{H}$ NMR (300 MHz, DMSO- $d_{6}$ ) $\delta(\mathrm{ppm}) 11.17$ (br $\mathrm{s}, 1 \mathrm{H},-\mathrm{NH}), 8.50(\mathrm{~s}, 1 \mathrm{H},-\mathrm{CH}=), 7.55(\mathrm{~d}, 2 \mathrm{H}$, $\left.{ }^{3} \mathrm{~J}=8.8 \mathrm{~Hz}, \mathrm{H}-3, \mathrm{H}-5\right), 7.39$ (d, 2H, ${ }^{3} \mathrm{~J}=8.8 \mathrm{~Hz}, \mathrm{H}-2$, H-6)

IR $\left(v, \mathrm{~cm}^{-1}\right) 3217,2225,2209,1668,811$

UV VIS (DMSO, nm) $\lambda_{\max } 330$

Elemental analysis for $\mathrm{C}_{10} \mathrm{H}_{6} \mathrm{BrN}_{3}$ - calculated (found) (\%): C 48.41 (48.35), H 2.44 (2.43), N $16.94(16.81)$

\section{Dimethyl 2-[(p-tolylamino $)$ methylidene]malonate (III $\mathrm{Z}, \mathrm{Z}=4-\mathrm{Me}$ )}

There was used to a reaction 4-methylaniline (Id, $\mathbf{Z}=\mathbf{4}$-Me $)(0.54 \mathrm{~g}, 5 \mathrm{mmol})$ in $8-10 \mathrm{ml} \mathrm{MeOH}$ and enolether IIa $(0.87 \mathrm{~g}, 5 \mathrm{mmol})$ in $8-10 \mathrm{ml} \mathrm{MeOH}$. Reaction mixture was refluxed under regular stirring for $3 \mathrm{~h}$. After its cooling raw product was recrystallized from petrolether.

Yield: $0.67 \mathrm{~g}, 54 \%$, colourless solid. Mp $104-106^{\circ} \mathrm{C}$ (108-110 ${ }^{\circ} \mathrm{C}$, Rappoport et al. 1972).

${ }^{1} \mathbf{H}$ NMR (400 MHz, $\mathrm{CDCl}_{3}$ ) $\delta$ (ppm) 11.01 (br s, 1H, $-\mathrm{NH}), 8.52\left(\mathrm{~d}, 1 \mathrm{H},{ }^{3} \mathrm{~J}=13.6 \mathrm{~Hz},-\mathrm{CH}=\right), 7.55(\mathrm{~d}$, $\left.2 \mathrm{H},{ }^{3} \mathrm{~J}=8.9 \mathrm{~Hz}, \mathrm{H}-2, \mathrm{H}-6\right), 7.17$ (d, $2 \mathrm{H},{ }^{3} \mathrm{~J}=8.4 \mathrm{~Hz}$, H-3, H-5), 3.86 (s, 3H, OMe), 3.78 (s, 3H, OMe), $2.34(\mathrm{~s}, 3 \mathrm{H}, \mathrm{Me})$

UV VIS (DMSO, nm) $\lambda_{\max } 330$

Elemental analysis for $\mathrm{C}_{13} \mathrm{H}_{15} \mathrm{NO}_{4}-$ calculated (found) (\%): C 62.64 (62.69), H 6.07 (5.96), N $5.62(5.44)$

\section{Diethyl 2-[(p-tolylamino $)$ methylidene]malonate \\ (IIIb, $Z=4-M e$ )}

There was used to a reaction 4-methylaniline (Id, $\mathbf{Z}=4$-Me) $(0.54 \mathrm{~g}, 5 \mathrm{mmol})$ in $8-10 \mathrm{ml} \mathrm{EtOH}$ and enolether IIb (1.1 g, $5 \mathrm{mmol})$ in 8-10 ml EtOH. Reaction mixture was refluxed under regular stirring for $3 \mathrm{~h}$. After its cooling solvent was evaporated and a raw product was purified by column chromatography $\left(\mathrm{EA}: \mathrm{H}=1: 1, \mathrm{R}_{\mathrm{F}}=0.20\right)$.

Yield: $1.31 \mathrm{~g}, 93 \%$, colourless solid. Mp $46-48^{\circ} \mathrm{C}$ (45-46 ${ }^{\circ} \mathrm{C}$, Mao et al. 2009).

${ }^{1} \mathbf{H}$ NMR $\left(300 \mathrm{MHz}^{\mathrm{CDCl}}{ }_{3}\right) \delta(\mathrm{ppm}) 11.00(\mathrm{~d}, 1 \mathrm{H}$, $\left.{ }^{3} J=13.6 \mathrm{~Hz},-\mathrm{NH}\right), 8.52\left(\mathrm{~d}, 1 \mathrm{H},{ }^{3} J=13.8 \mathrm{~Hz}\right.$, $-\mathrm{CH}=), 7.18\left(\mathrm{~d}, 2 \mathrm{H},{ }^{3} \mathrm{~J}=8.3 \mathrm{~Hz}, \mathrm{H}-2, \mathrm{H}-6\right)$, $7.05\left(\mathrm{~d}, 2 \mathrm{H},{ }^{3} \mathrm{~J}=8.4 \mathrm{~Hz}, \mathrm{H}-3, \mathrm{H}-5\right), 4.32$ (q, 2H, $\left.{ }^{3} J=7.1 \mathrm{~Hz}, \mathrm{OCH}_{2}\right), 4.26\left(\mathrm{q}, 2 \mathrm{H},{ }^{3} \mathrm{~J}=7.1 \mathrm{~Hz}\right.$, $\left.\mathrm{OCH}_{2}\right), 1.40\left(\mathrm{t}, 3 \mathrm{H},{ }^{3} \mathrm{~J}=7.1 \mathrm{~Hz}, \mathrm{CH}_{3}\right), 1.35(\mathrm{t}, 3 \mathrm{H}$, $\left.{ }^{3} J=7.1 \mathrm{~Hz}, \mathrm{CH}_{3}\right)$

UV VIS (DMSO, nm) $\lambda_{\max } 334$

\section{3-[(p-Tolylamino $)$ methylidene]pentane-2,4-dione}

(IIIc, $Z=4-M e$ )

There was used to a reaction 4-methylaniline (Id, $\mathbf{Z}=4$-Me) $(0.54 \mathrm{~g}, 5 \mathrm{mmol})$ in $10 \mathrm{ml} \mathrm{EtOH}$ and enolether IIc $(0.78 \mathrm{~g}, 5 \mathrm{mmol})$ in $20 \mathrm{ml} \mathrm{EtOH}$. Reaction mixture was stirred for $1 \mathrm{~h}$ at $\mathrm{rt}$. Solvent was evaporated and a raw product was collected by filtration and washed off with hexane.

Yield: $0.84 \mathrm{~g}, 77$ \%, light yellow cotton. Mp 136$138^{\circ} \mathrm{C}\left(139-140^{\circ} \mathrm{C}\right.$, Claisen 1893$)$.

${ }^{1} \mathbf{H}$ NMR $\left(300 \mathrm{MHz}^{\mathrm{CDCl}} 3\right.$ ) $\delta$ (ppm) 12.75 (br s, $1 \mathrm{H}$, $-\mathrm{NH}), 8.22\left(\mathrm{~d}, 1 \mathrm{H},{ }^{3} \mathrm{~J}=12.8 \mathrm{~Hz},-\mathrm{CH}=\right), 7.19(\mathrm{~d}$, $\left.2 \mathrm{H},{ }^{3} \mathrm{~J}=8.2 \mathrm{~Hz}, \mathrm{H}-2, \mathrm{H}-6\right), 7.08\left(\mathrm{~d}, 2 \mathrm{H},{ }^{3} \mathrm{~J}=8.4 \mathrm{~Hz}\right.$, $\mathrm{H}-3, \mathrm{H}-5), 2.57$ (s, 3H, 4-Me), 2.39 (s, 3H, Me), 2.37 (s, 3H, Me)

UV VIS (DMSO, nm) $\lambda_{\max } 345$

IR $\left(v, \mathrm{~cm}^{-1}\right) 2926,1595,1570,1308,814$

Elemental analysis for $\mathrm{C}_{13} \mathrm{H}_{15} \mathrm{NO}_{2}$ - calculated (found) (\%): C 71.87 (71.85), H 6.96 (6.91), N $6.45(6.32)$

\section{2-[(p-Tolylamino $)$ methylidene]malononitrile \\ (IIId, $Z=4-M e$ )}

There was used to a reaction 4-methylaniline (Id, $\mathbf{Z}=\mathbf{4}$-Me $)(0.54 \mathrm{~g}, 5 \mathrm{mmol})$ in $10 \mathrm{ml} \mathrm{EtOH}$ and enolether IId $(0.61 \mathrm{~g}, 5 \mathrm{mmol})$ in $20 \mathrm{ml} \mathrm{EtOH}$. Reaction mixture was stirred for $40 \mathrm{~min}$ at $\mathrm{rt}$. Solvent was evaporated and a raw product was washed off with cold EtOH.

Yield: $0.71 \mathrm{~g}, 76 \%$, yellow crystalls. Mp 289-290 ${ }^{\circ} \mathrm{C}$ (297 ${ }^{\circ} \mathrm{C}$, Rappoport et al. 1972).

${ }^{1} \mathbf{H}$ NMR (300 MHz, DMSO-d $\left.d_{6}\right) \delta(\mathrm{ppm}) 11.06(\mathrm{br}$ $\mathrm{s}, 1 \mathrm{H},-\mathrm{NH}), 8.40(\mathrm{~s}, 1 \mathrm{H},-\mathrm{CH}=), 7.27(\mathrm{~d}, 2 \mathrm{H}$, 
$\left.{ }^{3} \mathrm{~J}=8.4 \mathrm{~Hz}, \mathrm{H}-2, \mathrm{H}-6\right), 7.14\left(\mathrm{~d}, 2 \mathrm{H},{ }^{3} \mathrm{~J}=8.4 \mathrm{~Hz}, \mathrm{H}-3\right.$, $\mathrm{H}-5), 2.24$ (s, 3H, Me)

UV VIS (DMSO, nm) $\lambda_{\text {max }} 320$

Elemental analysis for $\mathrm{C}_{11} \mathrm{H}_{9} \mathrm{~N}_{3}-$ calculated (found) (\%): 72.11 (72.02), H 4.95 (4.88), N $22.94(22.89)$

\section{Dimethyl 2-[(4-methoxyphenylamino)methylidene]} malonate (IIIa, $\mathrm{Z}=4-\mathrm{OM}$ )

There was used to a reaction 4-methoxyaniline (Ie, $\mathbf{Z}=$ 4-OMe) $(0.62 \mathrm{~g}, 5 \mathrm{mmol})$ in $15 \mathrm{ml} \mathrm{MeOH}$ and enolether IIa $(0.87 \mathrm{~g}, 5 \mathrm{mmol})$ in $15-20 \mathrm{ml} \mathrm{MeOH}$. Reaction mixture was refluxed under regular stirring for $3 \mathrm{~h}$. After its cooling raw product was recrystallized from petrolether.

Yield: $0.64 \mathrm{~g}, 48 \%$, colourless solid. Mp 86-89 ${ }^{\circ} \mathrm{C}$ (89-91 ${ }^{\circ} \mathrm{C}$, Zewge et al. 2007).

${ }^{1} \mathbf{H}$ NMR (400 $\left.\mathrm{MHz} \mathrm{CDCl}_{3}\right) \delta(\mathrm{ppm}) 11.02(\mathrm{br}$ $\mathrm{s}, 1 \mathrm{H},-\mathrm{NH}), 8.45\left(\mathrm{~d}, 1 \mathrm{H},{ }^{3} \mathrm{~J}=8.7 \mathrm{~Hz},-\mathrm{CH}=\right)$, $7.11-6.89(\mathrm{~m}, 4 \mathrm{H}), 3.86$ (s, 3H, OMe), 3.81 (s, 3H, OMe), 3.78 (s, 3H, 4-OMe)

IR $\left(v, \mathrm{~cm}^{-1}\right) 2946,1683,1645,1449,1266,1241,797$ UV VIS (DMSO, nm) $\lambda_{\max } 324$

Elemental analysis for $\mathrm{C}_{13} \mathrm{H}_{15} \mathrm{NO}_{5}$ - calculated (found) (\%): C 58.86 (58.74), H 5.70 (5.65), N $5.28(5.12)$

Diethyl 2-[(4-methoxyphenylamino)methylidene]malonate (IIIb, $Z=4-O M e)$

There was used to a reaction 4-methoxyaniline (Ie, $\mathbf{Z}=$ 4-OMe) $(0.62 \mathrm{~g}, 5 \mathrm{mmol})$ in $10 \mathrm{ml} \mathrm{MeOH}$ and enolether IIb (1.1 g, $5 \mathrm{mmol})$ in $20 \mathrm{ml} \mathrm{EtOH}$. Reaction mixture was refluxed under regular stirring for $3 \mathrm{~h}$. After its cooling raw product was purified by column chromatography $\left(\mathrm{EA}: \mathrm{H}=10: 1, \mathrm{R}_{\mathrm{F}}=0.20\right)$.

Yield: $1.22 \mathrm{~g}, 82 \%$, orange solid. $\mathrm{Mp}<35^{\circ} \mathrm{C}$ (38-39 ${ }^{\circ} \mathrm{C}$, Deshmukh et al. 1999).

${ }^{1} \mathbf{H}$ NMR (300 MHz, $\left.\mathrm{CDCl}_{3}\right) \delta$ (ppm) 10.95 (br s, 1H, $-\mathrm{NH}), 8.40\left(\mathrm{~d}, 1 \mathrm{H},{ }^{3} \mathrm{~J}=13.8 \mathrm{~Hz},-\mathrm{CH}=\right), 7.04(\mathrm{~d}$, $\left.2 \mathrm{H},{ }^{3} \mathrm{~J}=8.7 \mathrm{~Hz}, \mathrm{H}-2, \mathrm{H}-6\right), 6.86\left(\mathrm{~d}, 2 \mathrm{H},{ }^{3} \mathrm{~J}=8.7 \mathrm{~Hz}\right.$, $\mathrm{H}-3, \mathrm{H}-5), 4.31-4.17\left(\mathrm{~m}, 4 \mathrm{H}, \mathrm{OCH}_{2}\right), 3.76(\mathrm{~s}, 3 \mathrm{H}$, OMe), 1.37-1.27 (m, 6H, $\left.\mathrm{CH}_{3}\right)$

UV VIS (DMSO, nm) $\lambda_{\max } 321$

\section{2-[(4-Methoxyphenylamino) methylidene]pentane-2, 4-} dione (IIIc, $Z=4$-OMe)

There was used to a reaction 4-methoxyaniline (Ie, $\mathbf{Z}=\mathbf{4 - O M e})(0.62 \mathrm{~g}, 5 \mathrm{mmol})$ in $10 \mathrm{ml} \mathrm{EtOH}$ and enolether IIc $(0.78 \mathrm{~g}, 5 \mathrm{mmol})$ in $20 \mathrm{ml} \mathrm{EtOH}$. Reaction mixture was stirred for $1 \mathrm{~h}$ at $\mathrm{rt}$. Solvent was evaporated and a raw product was purified by column chromatography $\left(\mathrm{EA}: \mathrm{H}=1: 1, \mathrm{R}_{\mathrm{F}}=0.31\right)$.

Yield: $1.09 \mathrm{~g}, 95 \%$, light yellow solid. Mp 85$87^{\circ} \mathrm{C}$.

${ }^{1} \mathbf{H}$ NMR (300 MHz, $\left.\mathrm{CDCl}_{3}\right) \delta$ (ppm) 12.79 (br s, 1H, $-\mathrm{NH}), 8.15\left(\mathrm{~d}, 1 \mathrm{H},{ }^{3} \mathrm{~J}=12.8 \mathrm{~Hz},-\mathrm{CH}=\right), 7.12(\mathrm{~d}$, $\left.2 \mathrm{H},{ }^{3} \mathrm{~J}=8.9 \mathrm{~Hz}, \mathrm{H}-2, \mathrm{H}-6\right), 6.93\left(\mathrm{~d}, 2 \mathrm{H},{ }^{3} \mathrm{~J}=8.9 \mathrm{~Hz}\right.$, H-3, H-5), 3.83 (s, 3H, OMe), 2.55 (s, 3H, Me), $2.37(\mathrm{~s}, 3 \mathrm{H}, \mathrm{Me})$

IR $\left(v, \mathrm{~cm}^{-1}\right)$ 2990, 1616, 1594, 1318, 1249, 979, 828

UV VIS (DMSO, nm) $\lambda_{\max } 342$

Elemental analysis for $\mathrm{C}_{13} \mathrm{H}_{15} \mathrm{NO}_{3}$ - calculated (found) (\%): C 66.94 (66.78), H 6.48 (6.37), N $6.00(5.92)$

\section{2-[(4-metoxyphenylamino)methylidene]malononitrile}

(IIId, $Z=4-O M e$ )

There was used to a reaction 4-methoxyaniline (Ie, $\mathbf{Z}=\mathbf{4}$-OMe) $(0.62 \mathrm{~g}, 5 \mathrm{mmol})$ in $10 \mathrm{ml} \mathrm{EtOH}$ and enolether IId $(0.61 \mathrm{~g}, 5 \mathrm{mmol})$ in $15 \mathrm{ml} \mathrm{EtOH}$. Reaction mixture was stirred for $30 \mathrm{~min}$ at rt. Raw product was collected by filtration and washed off with hexane.

Yield: $0.70 \mathrm{~g}, 70 \%$, light yellow powder. Mp 266-267 ${ }^{\circ} \mathrm{C}\left(245^{\circ} \mathrm{C}\right.$, Rappoport et al. 1972).

${ }^{1}$ H NMR (300 MHz, DMSO- $\left.d_{6}\right) \delta(\mathrm{ppm}) 11.03$ (br $\mathrm{s}, 1 \mathrm{H},-\mathrm{NH}), 8.37(\mathrm{~s}, 1 \mathrm{H},-\mathrm{CH}=), 7.35(\mathrm{~d}, 2 \mathrm{H}$, $\left.{ }^{3} J=9.0 \mathrm{~Hz}, \mathrm{H}-2, \mathrm{H}-6\right), 6.93$ (d, 2H, ${ }^{3} \mathrm{~J}=9.0 \mathrm{~Hz}, \mathrm{H}-3$, $\mathrm{H}-5), 3.74$ (s, 3H, OMe)

UV VIS (DMSO, nm) $\lambda_{\max } 335$

Elemental analysis for $\mathrm{C}_{11} \mathrm{H}_{9} \mathrm{~N}_{3} \mathrm{O}$ - calculated (found) (\%): C 66.32 (66.41), H 4.55 (4.49), N 21.09 (21.06)

\section{Dimethyl 2-[(4-nitrophenylamino)methylidene]malonate} (IIIa, $\mathrm{Z}=4-\mathrm{NO}_{2}$ )

There was used to a reaction 4-nitroaniline (If, $\left.\mathbf{Z}=\mathbf{4}-\mathbf{N} \mathbf{O}_{2}\right)(0.69 \mathrm{~g}, 5 \mathrm{mmol})$ in $10 \mathrm{ml} \mathrm{MeOH}$ and enolether IIa $(0.87 \mathrm{~g}, 5 \mathrm{mmol})$ in $20 \mathrm{ml} \mathrm{MeOH}$. Reaction mixture was refluxed under regular stirring for $3 \mathrm{~h}$. After its cooling raw product was collected by filtration and washed off with cold $\mathrm{MeOH}$.

Yield: $0.91 \mathrm{~g}, 65 \%$, yellow crystalline product. Mp $156-157^{\circ} \mathrm{C}$.

${ }^{1}$ H NMR (400 MHz, $\mathrm{CDCl}_{3}$ ) $\delta$ (ppm) 11.23 (br s, $1 \mathrm{H},-\mathrm{NH}), 8.54$ (br s, $1 \mathrm{H},-\mathrm{CH}=), 8.25(\mathrm{~d}, 2 \mathrm{H}$, $\left.{ }^{3} J=9.1 \mathrm{~Hz}, \mathrm{H}-3, \mathrm{H}-5\right), 7.62$ (d, 2H, ${ }^{3} J=9.1 \mathrm{~Hz}, \mathrm{H}-2$, H-6), 3.88 (s, 3H, OMe), 3.82 (s, 3H, OMe)

UV VIS (DMSO, nm) $\lambda_{\max } 367$

Elemental analysis for $\mathrm{C}_{12} \mathrm{H}_{12} \mathrm{~N}_{2} \mathrm{O}_{6}-$ calculated (found) (\%): C 51.43 (51.35), H 4.32 (4.28), N $10.00(9.90)$

Diethyl 2-[(4-nitrophenylamino)methylidene]malonate (IIIb, $\mathrm{Z}=4-\mathrm{NO}_{2}$ )

There was used to a reaction 4-nitroaniline (If, $\left.\mathbf{Z}=\mathbf{4}-\mathbf{N O}_{\mathbf{2}}\right)(0.69 \mathrm{~g}, 5 \mathrm{mmol})$ in $10 \mathrm{ml} \mathrm{EtOH}$ and enolether IIb (1.1 g, $5 \mathrm{mmol})$ in $20 \mathrm{ml} \mathrm{EtOH.} \mathrm{Reac-}$ tion mixture was refluxed under regular stirring for $3 \mathrm{~h}$. After its cooling raw product was collected by filtration and washed off with cold EtOH. 
Yield: 1.13 g, $72 \%$, yellow solid. Mp $136-138{ }^{\circ} \mathrm{C}$ (142 ${ }^{\circ} \mathrm{C}$, Darque et al. 2009).

${ }^{1} \mathbf{H}$ NMR (400 MHz, $\left.\mathrm{CDCl}_{3}\right) \delta$ (ppm) 11.20 (br s, $1 \mathrm{H},-\mathrm{NH}), 8.51(\mathrm{~s}, 1 \mathrm{H},-\mathrm{CH}=), 8.20(\mathrm{~d}, 2 \mathrm{H}$, $\left.{ }^{3} \mathrm{~J}=9.2 \mathrm{~Hz}, \mathrm{H}-3, \mathrm{H}-5\right), 7.60$ (d, 2H, ${ }^{3} \mathrm{~J}=9.2 \mathrm{~Hz}, \mathrm{H}-2$, H-6), 4.23 (q, 2H, $\left.{ }^{3} J=7.1 \mathrm{~Hz}, \mathrm{OCH}_{2}\right), 4.15$ (q, 2H, $\left.{ }^{3} J=7.1 \mathrm{~Hz}, \mathrm{OCH}_{2}\right), 1.28\left(\mathrm{t}, 3 \mathrm{H},{ }^{3} J=7.0 \mathrm{~Hz}, \mathrm{CH}_{3}\right)$, $1.24\left(\mathrm{t}, 3 \mathrm{H},{ }^{3} \mathrm{~J}=7.0 \mathrm{~Hz}, \mathrm{CH}_{3}\right)$

UV VIS (DMSO, nm) $\lambda_{\max } 373$

\section{Results and discussion}

To avoid geometrical isomerism on the double bond of the products of the nucleophilic vinylic substitution (Saloň et al. 2005) and therefore thus influence the kinetics of this reaction, we decided to prepare only compounds with the same substituents on the $\beta$ - position of the double bond, e.g. on the opposite side to arylamino substituent. Therefore, reaction of the dimethyl methoxymethylidene malonate (IIa), diethyl ethoxymethylidene malonate (IIb), ethoxymethylidene pentane-2,4-dione (IIc) and ethoxymethylidene malononitrile (IId) respectively, with the appropriate substituted aniline in the same alcohol like alkoxy and/or ester group was used for the preparation of target compounds.

\section{NMR spectra and the substituent chemical shifts (SCS) of the analogous anilinomethylidene (nonsubstituted) compounds}

In 1989 Goljer and co-workers published carbon spectra of the appropriate anilinomethylidene (nonsubstituted) compounds IV (equal to III: $\mathrm{Z}=\mathrm{H}$ ) (Tab. 1, 2).

\section{NMR spectra and the substituent chemical shifts} (SCS) of the studied compounds

Applying of the published substituent chemical shifts (Ewing 1979) of the substituents of the benzene ring regarding the position of the substituent it is possible to calculate the chemical shifts of the studied compounds and compare them with measured ones to assign them. Of course, there exist some other methods like the use of plugins of the drawing programs like ISIS Draw ${ }^{\circledR}$, ChemDraw ${ }^{\circledR}$ etc., but the used algoritm is not clear. Another alternative is the calculation using semiempirical/ ab initio calculation methods.

In the Table 3 there are presented all our recorded ${ }^{13} \mathrm{C}$ NMR spectra in first row for each compound. In next row $(\mathrm{s})$ there are presented calculated values obtained using appropriate values for aminoethylidene substituent in deuterochloroform (Table 3)/ hexadeuterodimethyl sulfoxide (Table 1) and for the appropriate substituent in right position and solvent. In all cases we used also data for neat liquid (NL). In the column Reference, there are references for the work, where the spectra of the corresponding compounds were appeared first.

Substituent chemical shifts are frequently used in proton and carbon spectra for founding of the unknown values of the chemical shifts of the new synthesized substances to confirm their structure. Some of them are used like sole, some should be combined. We selected the second approach, e.g. combine SCS for monosubstituted benzenes to obtain set of datas for disubstituted benzenes. Data for proton spectra are more sensitive for solvents, for example dimethyl sulfoxide (Rao et al. 1974). Carbon data reflecting well electron distribution in the molecule and therefore SCS are in relation with electron properties of the substituents.

$\beta, \beta$-Disubstituted aminoethylidene substituent with two electron withdrawing groups displayed an interesting influence on benzene ring: on ipso carbon it has practically the same values like on ortho car-

Tab. 1. ${ }^{13} \mathrm{C}$ chemical shifts of the studied compounds IV in DMSO-d $\mathrm{d}_{6}$.

\begin{tabular}{cccccccc}
\hline Compound & C - 1 & C - 2 & C - - & C - - & C - 7 & C - 8 & Others \\
\hline IVa & 139.3 & 117.6 & 129.6 & 124.7 & 151.4 & 92.6 & $165.2,167.5,51.0,50.9$ \\
IVb & 139.3 & 117.3 & 129.6 & 124.6 & 150.9 & 93.6 & $165.0,167.4$ \\
IVc & 139.0 & 118.1 & 129.3 & 125.3 & 155.5 & 52.0 & $116.3,113.9$ \\
IVd & 139.0 & 118.2 & 129.5 & 125.2 & 152.6 & 112.4 & $195.1,199.4,27.4,31.4$ \\
\hline
\end{tabular}

Tab. 2. Substituent chemical shifts of the studied compounds IV in DMSO-d . $_{\text {. }}$

\begin{tabular}{ccccc}
\hline Compound & G - 1 & G - 2 & C - 3 & G - 4 \\
\hline IVa & +10.5 & -10.3 & +1.0 & -3.3 \\
IVb & +10.5 & -10.4 & +0.8 & -3.2 \\
IVc & +10.8 & -10.9 & +1.1 & -3.8 \\
IVd & +10.8 & -11.2 & +1.1 & -3.9 \\
\hline
\end{tabular}




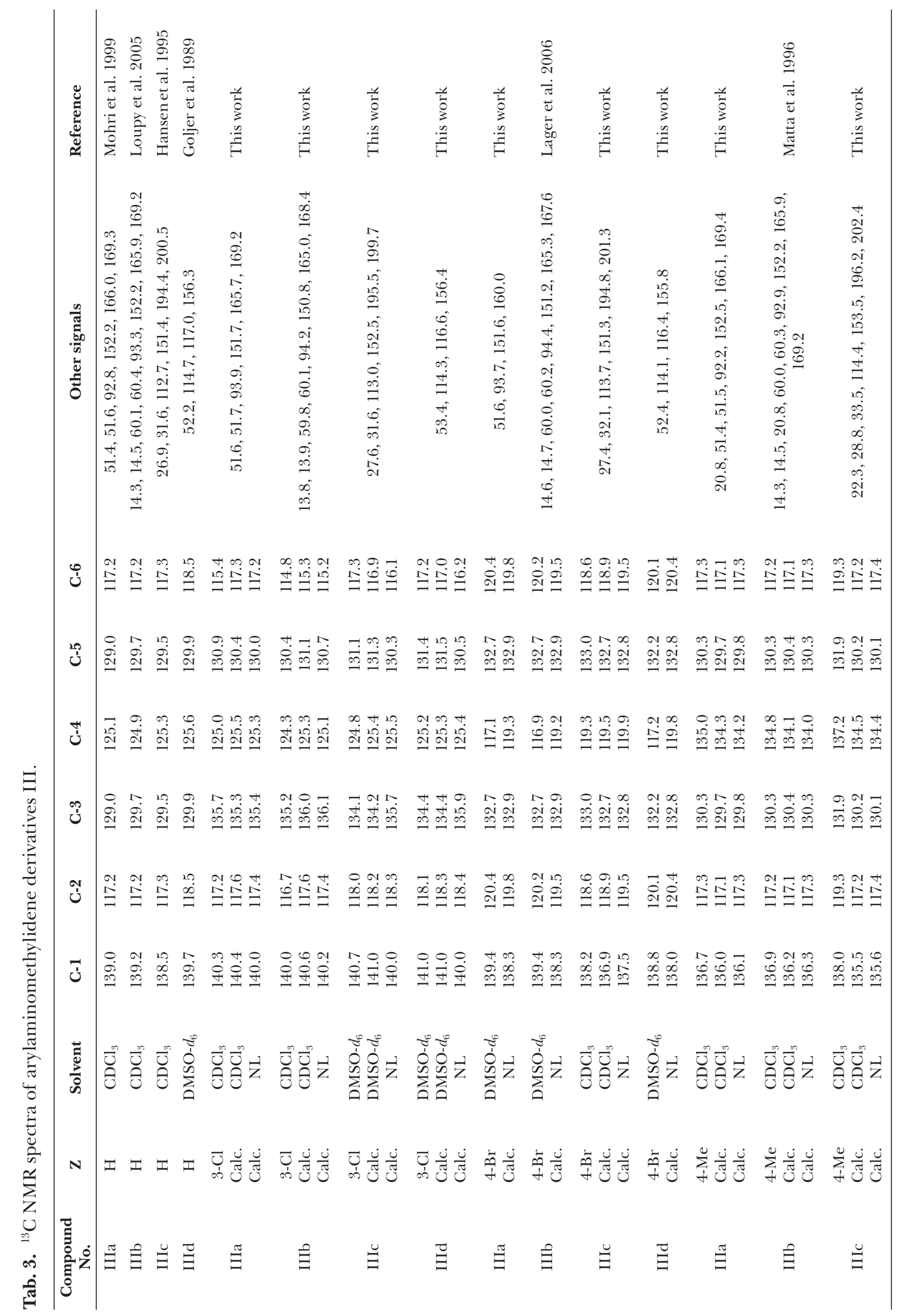




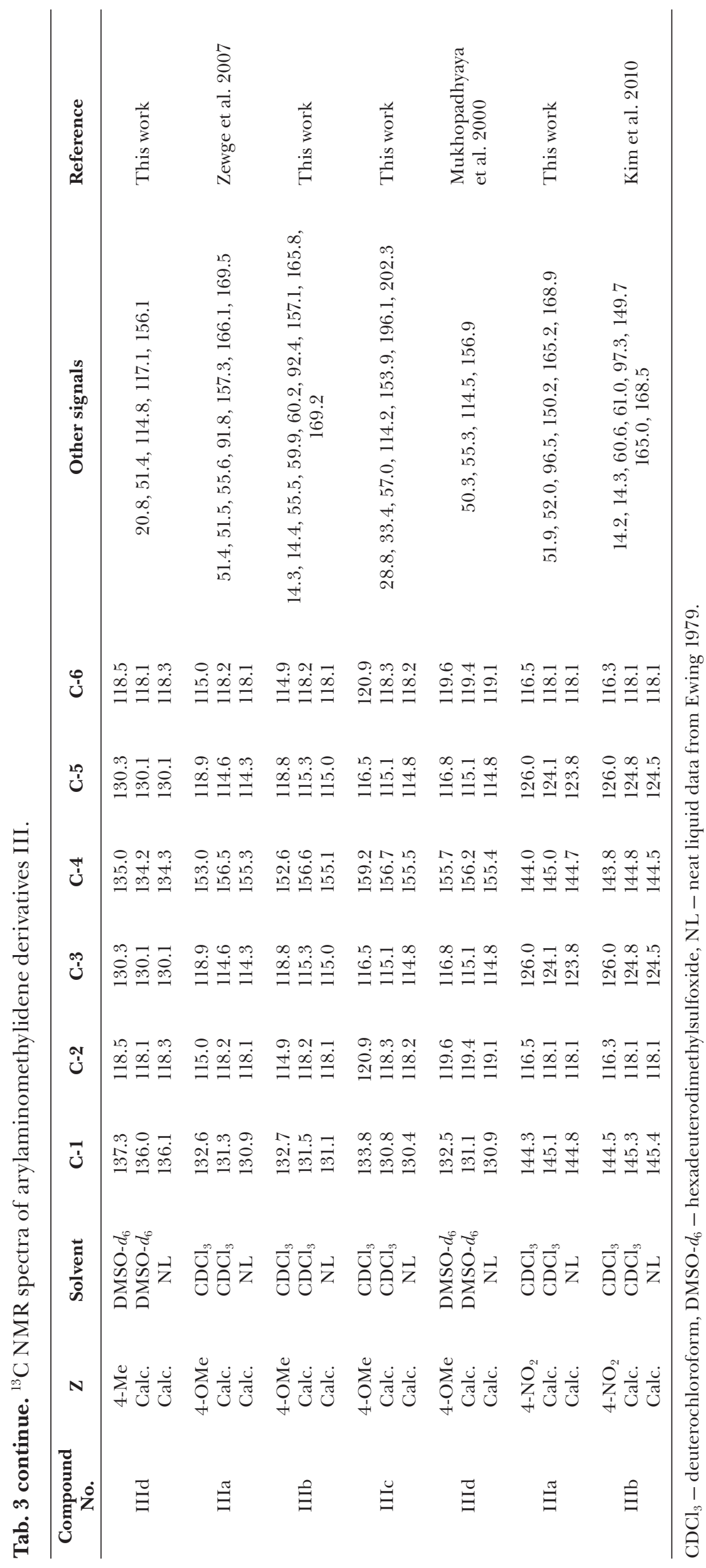


bons, but reversal sign. It is not so strong effect like the amino group, but slighly similar to aminoacetyl group, thus similar to aminogroup substituted with polarized double bond. The obtained data are in good agreement with calculated ones within small range of deviations what is confirming the accuracy of the used method. On the other hand, meta- and para-substitution does not twisting the aminoethylidene substituent off of the plane of the benzene ring. In Table 3 are presented ${ }^{13} \mathrm{C}$ NMR data of the products III obtained from nucleophilic vinylic substitution of substituted anilines I and activated enolethers II.

\section{Acknowledgement}

This work was supported by the Slovak Research and Development Agency under contracts Nos APPV-0338-11 and APVV-0339-10, and by the Scientific Grant Agency of the Slovak Republic (Projects VEGA 1/0660/11). The NMR experimental part of this work was facilitated by the support of Slovak National Research and Development Program No. 2003SP200280203. Authors acknowledging Dr. N. Prónayouá for recording NMR spectra.

\section{References}

Bella M, Schultz M, Milata V (2012) Arkivoc IV: 242251.

Claisen L (1893) Chem. Ber. 26: 2729-2735.

Couchouron B, Le Saint J, Courtot P (1983) Bull. Soc. Chim. Fr. II: 66-71.

Darque A, Dumetre A, Hutter S, Azas N, Casano G, Robin M, Pannecouque Ch (2009) Bioorg. Med. Chem. Lett. 19: 5962-5964.

Deshmukh ARAS, Panse DG, Bhawal BM (1999) Synth. Commun. 29: 1801-1810.

Ewing DF (1979) Org. Magn. Reson. 12: 499-524.

Fodor G, Wein J, Schönberg A, Duffin GF, Kendall JD, Seetharamiah A (1948) J. Chem. Soc. 890-895.

Goljer I, Milata V, Ilavský D (1989) Magn. Reson. Chem. 27: 138-144.

Hansen PE, Bolvig S, Duus F, Petrova MV, Kawecki R, Krajewski R, Kozerski L (1995) Magn. Reson. Chem. 33: 621-631.
Hermecz I, Keresztúri G, Vasvári-Debreczy L (1992) in Advances in Heterocyclic Chemistry, Vol. 54: Aminomethylenemalonates and Their Use in Heterocyclic Synthesis, ed. A.R. Katritzky, Academic Press, San Diego, 452 p.

Huppatz JL, Phillips JN, Rattigan BM (1981) Agricult. Biol. Chem., 45: 2769-2774.

Kim KW, Lee HJ, Jo JI, Kwon, TW (2010) Bull. Korean Chem. Soc. 31: 1155-1158.

Lager E, Andersson P, Nilsson J, Pettersson I, Nielsen EO, Nielsen M, Sterner O, Liljefors T (2006) J. Med. Chem. 49: 2526-2533.

Lopez R, Marisa J, Moyano EL, Yranzo GI (2010) Tetrahedron Lett. 51: 478-481.

Loupy A, Song SJ, Cho SJ, Park DK, Kwon TW (2005) Synth. Commun. 35: 79-88.

Mao D, Xu J, Hu X, Dong J, Zhang G, Gong G (2009) Chem. Biodiv. 6: 1727-1736.

da Matta AD, Bernardino AMR, Romeiro GA, de Oliveira MRP, Souza MCBV, deFerreira VF (1996) Nucleosides \& Nucleotides 15: 889-898.

Milata V, Ilavský D (1987) Coll. Czechoslov. Chem. Commun. 52: 2918-2925.

Mohri K, Kanie A, Horiguchi Y, Isobe K (1999) Heterocycles 51: 2377-2384.

Mukhopadhyaya JK, Sklenak S, Rappoport Z (2000) J. Org. Chem. 65: 6856-6867.

Nasakin O, Lyshchikov AN, Lukin PM, Tafeenko VA, Bulai AKh, Medvedev SV (1992) Chem. Het. Comp. 28: $1124-1129$.

Rao V, Balakrishnan M, Venkatasubramanian N (1974) Indian J. Chem. 13: 1090-1091.

Rappoport Z, Topol A (1972) J. Chem. Soc. Perkin Trans. 2: $1823-1831$.

Saloň J, Milata V, Gatial A, Prónayová N, Leško J, Černuchová P, Rappoport Z, Vo-Thanh G, Loupy A (2005) Eur. J. Org. Chem. 22: 4870-4878.

Santilli AA (1964) J. Med. Chem. 7: 68-72.

Snyder J, Jones RE (1946) J. Am. Chem. Soc. 68: 1253-1255.

Wolfbeis OS (1977) Synth. 723-725.

Wolfbeis OS, Junek HZ (1979) Naturforsch. [B] 34: 283-286.

Zewge D, Chen Ch-Y, Deer C, Dormer PG, Hughes DL (2007) J. Org. Chem. 72: 4276-4279. 